Gónzalez-Gil, F., Martín-Pastor, E., Poy, R. \& Jenaro, C. (2016). Percepciones del profesorado sobre la inclusión: estudio preliminar. Revista Electrónica Interuniversitaria de Formación del Profesorado, 19(3), 11-24.

\title{
Percepciones del profesorado sobre la inclusión: estudio preliminar
}

\author{
Francisca González-Gil*, Elena Martín-Pastor*, Raquel Poy**, Cristina Jenaro*** \\ *Facultad de Educación (Universidad de Salamanca), **Facultad de Educación (Universidad
} de León), ***Facultad de Psicología (Universidad de Salamanca)

\section{Resumen}

Todo sistema educativo debe tener como prioridad la atención y respuesta a las diferentes necesidades de sus alumnos, potenciando sus capacidades y facilitando la adquisición de los aprendizajes establecidos para cada etapa educativa. Numerosos estudios consideran al profesorado una pieza clave en este proceso, lo que nos lleva a reflexionar sobre el grado en que los docentes se encuentran preparados para asumir este reto. Así, el objetivo del presente estudio es analizar las actitudes y las necesidades formativas de los docentes respecto a las culturas, políticas y prácticas inclusivas. Para ello se elaboró un cuestionario ad hoc que fue aplicado a 402 profesionales de la educación de Castilla y León. Los resultados muestran la existencia de necesidades relacionadas en general con la transformación de las escuelas en centros educativos inclusivos, y en particular, con la formación en metodologías de trabajo más inclusivas, y estrategias para abordar todo el proceso.

\section{Palabras clave}

Formación docente; actitudes; culturas, políticas y prácticas inclusivas.

\section{Contacto:}

Elena Martín-Pastor, emapa@usal.es, Facultad de Educación, Paseo de Canalejas, 169, 37008, Salamanca.

Consejería de Educación de la Junta de Castilla y León (Proyecto SA004B10-1). 


\title{
Teachers' perceptions of inclusion: preliminary study
}

\begin{abstract}
An inherent feature of the current society and, therefore, of the educational context is the diversity. This requires the need of an education that responds to the needs of each student, strengthen capabilities and make easier the learning process. All of this under the paradigm of inclusion. That justifies the need of reflect on the degree that teachers are trained to assume this challenge. The present study aims to analyze teachers 'attitudes and training needs regarding inclusive cultures, policies and practices. To test this, a sample of 402 teachers from Castilla y León was selected. An ad hoc measure, developed for the current study, was used for the assessment. Results revealed high training needs for education professionals, which are mostly related to the lack of adequate training to participate in transforming their schools into inclusive settings, and with a lack of inclusive methodologies for implementation in schools.
\end{abstract}

\section{Key words}

Training of teachers; attitudes; inclusive cultures, politics and practices.

\section{Introducción}

Colectivos muy diversos de nuestra sociedad han sido, y en ocasiones siguen siéndolo, objeto de exclusión: personas con discapacidad, mujeres, minorías étnicas, grupos culturales minoritarios, clases sociales marginales, inmigrantes, etc. Al trasladar lo anterior al contexto educativo se pone de manifiesto la necesidad de construir una escuela para todos donde la diversidad se perciba como una oportunidad y no como una amenaza (López Melero, 2012, Sapon Shevin, 2013). En este sentido, no podemos olvidar que en última instancia es cada centro educativo quien a través de sus estructuras, su relaciones, sus códigos y prácticas específicas, etiqueta y determina quiénes son alumnos "normales" y quienes son "diversos", la que les plantea unas demandas y exigencias, les brinda o no ciertos apoyos y acogidas y, en definitiva, los sitúa en una cultura escolar más o menos inclusiva (González, 2008).

Como refleja Blanco (2008), la educación inclusiva implica una visión diferente de la educación basada en la diversidad y no en la homogeneidad. Así, su foco de actuación es la transformación de los sistemas educativos, mediante la modificación de tres elementos fundamentales (Booth y Ainscow, 2002, 2011; Booth, Ainscow y Kingston, 2006): sus culturas, sus prácticas educativas y sus políticas para que atiendan la diversidad de necesidades educativas del alumnado, y lograr con ello el pleno aprendizaje y participación de cada niño (Blanco, 2008), desde el convencimiento de que la educación no mejorará, a menos que los sistemas escolares asuman que todos los alumnos son competentes para aprender y a menos que se reduzcan las desigualdades en contenidos, oportunidades y resultados (Escudero y Martínez, 2012; López Melero, 2012).

Si bien, desde diversos frentes se está apostando por la inclusión como modelo y se están realizando esfuerzos para delimitar los indicadores cuantitativos y cualitativos de la educación inclusiva (Göransson y Nilholm, 2014; Kyriazopoulou y Weber, 2009), queda aún un amplio camino por recorrer. A este respecto, Álvarez, Álvarez, Castro, Campo y Fueyo 
(2008) ponen de manifiesto que los profesores consideran que el modelo integrador no responde a las necesidades de todos los alumnos y que las medidas desarrolladas no resultan todo lo eficaces que deberían. En relación con ello, Donnelly y Watkins (2011) o Torres y Fernández (2015), entre otros, destacan el papel decisivo que los profesores y los formadores de profesores juegan a la hora de abordar estos retos.

Existe un amplio consenso (Echeita, 2007; Echeita et al, 2008; Vlachou, 2004) en que para progresar en los modos de responder a las necesidades educativas de los alumnos no basta con apelar a la ética ni a los valores o a los potenciales efectos beneficiosos que ello reportaría al conjunto del sistema educativo. Tampoco es suficiente con presentar al profesorado nuevas concepciones o nuevos recursos o pautas de acción eficaces: es necesario modificar las creencias implícitas al respecto. De hecho, la principal barrera para conseguir que los centros escolares sean organizaciones atentas a la diversidad se encuentra en las ideas, las normas, las creencias y actitudes vigentes en la escuela, los patrones de funcionamiento y las prácticas de los agentes implicados (Ahmmed, Sharma y Deppeler, 2014; González, 2008; Weiß, Kollmannsberger, Lerche, Oubaid y Kiel, 2014). En la misma línea, Parrilla (2007) hace referencia a algunas prácticas educativas calificadas como inclusivas que, sin embargo, no hacen más que perpetuar el estatus quo del sistema y abrir nuevas puertas a la marginación y la desigualdad entre alumnos. Uno de los factores que más influyen en ello son las actitudes docentes como predictoras de la aceptación de la diversidad, influyendo además en la construcción del conocimiento del profesorado sobre sus alumnos, la enseñanza y su propia práctica docente, configurándose de este modo como un aspecto clave en las expectativas que desarrollan sobre los estudiantes y sus aprendizajes, y que al mismo tiempo se relacionan con el rendimiento académico del alumnado (Aguado, Alcedo y Arias, 2008; Alemany y Villuendas, 2004; Álvarez, Castro, Campo y Álvarez, 2005; Avramidis y Kalyra, 2007; Chiner, 2011; López López y Hinojosa, 2012; Torres y Fernández, 2015).

Otro, la formación del profesorado para lograrlo. Echeita et ál. (2008) analizaron la inclusión educativa española en los últimos años, y concluyeron que la investigación y la experiencia apuntan, una y otra vez, a que un profesorado bien formado, competente, reflexivo y comprometido con los valores de la inclusión es la mejor garantía para poder identificar las barreras de distinto tipo que perviven en las culturas y en las prácticas de los centros escolares. Del mismo modo se manifiestan Sánchez Palomino (2007) o Mosia (2014) al señalar que bajos índices de formación docente en atención a la diversidad pueden propiciar actitudes negativas o de rechazo que repercutan en pobres estrategias de enseñanza-aprendizaje que respondan a una verdadera educación inclusiva. Sin embargo, éstos son aspectos que no han sido abordados en profundidad en la enseñanza y, por ende, constituyen una asignatura pendiente en la misma (López López y Hinojosa, 2012). A modo de ilustración, Molina y Holland (2010) recogen las palabras de Lambe y Bones (2006) para quienes la formación inicial de profesorado aparece como el método más efectivo para promover mejores actitudes hacia la inclusión. De hecho, la $48^{\text {a }}$ Conferencia Internacional de Educación de la UNESCO identificó la formación de profesores como la clave para el desarrollo futuro (Acedo, 2011). De hecho, el Informe McKinsey (2007) sobre los 25 mejores sistemas educativos concluyó que la principal variable que explica las diferencias en el aprendizaje de los alumnos es la calidad de los profesores. Por tanto, si los profesores no se sienten preparados para trabajar con todos los alumnos, el reto está en mejorar los programas de formación de maestros, dotando a los participantes de los recursos necesarios para lograrlo.

Una de las herramientas que favorece el desarrollo de contextos educativos inclusivos es el Index for Inclusion (Booth y Ainscow, 2002, 2011; Booth, Ainscow y Kingston, 2006), 
desarrollado en el mundo anglosajón, y que ha sido adaptado a numerosas lenguas, incluyendo el castellano (González-Gil, Gómez-Vela y Jenaro, 2007; López, Durán, Echeita, Giné, Miquel y Sandoval, 2002). Dicha herramienta permite evaluar el grado de inclusión de los centros educativos a partir de sus culturas, políticas y prácticas para, a partir de los resultados obtenidos, promover mejoras en cada una de estas dimensiones. Varios estudios mostraron en su momento la eficacia de su uso (Durán, Echeita, Giné, Miquel, Ruiz y Sandoval, 2005; Miquel, 2009; Booth, 2009), y la actualización permanente de las versiones del Index avala la continuidad y la utilidad de su aplicación.

Por todo lo expuesto y teniendo en cuenta la importancia del tema y la escasez de trabajos realizados desde un planteamiento inclusivo, presentamos a continuación un estudio cuyo objetivo ha sido analizar las actitudes y las necesidades formativas de los docentes tomando como referencia las tres dimensiones que establece el Index for Inclusion: crear culturas inclusivas, generar políticas inclusivas y desarrollar prácticas inclusivas (Booth y Ainscow, 2002, 2011; Booth, Ainscow y Kingston, 2006). Esperamos además, en consonancia con estudios previos, encontrar diferencias en la percepción de la inclusión en función de variables como: género (Almeida y Albert, 2009; Chiner, 2011), años de experiencia docente (Álvarez et ál., 2008; Chiner, 2011; Colmenero, 2009), formación en educación (Alemany y Villuendas, 2004; Almeida y Albert, 2009; Álvarez et ál., 2005; Avramidis y Kalyra, 2007; Chiner, 2011; Jurado de los Santos y Olmos Rueda, 2010; Lledó y Arnáiz, 2010; Sánchez Palomino, 2007) y titularidad de centro (Álvarez et ál., 2008; Jurado de los Santos y Olmos Rueda, 2010; Lledó y Arnáiz, 2010).

\section{Método}

Se trata de un estudio descriptivo-correlacional y con medidas ex post facto.

\section{Participantes}

La población objeto de estudio la componen los maestros que imparten docencia en los centros educativos de la comunidad autónoma de Castilla y León en las etapas de educación infantil, primaria y secundaria. Concretamente, la muestra estuvo formada por 402 profesores, los cuales aceptaron participar en este estudio de manera voluntaria. En todo momento se garantizó el anonimato y la confidencialidad de los datos. Un $63,7 \%$ eran mujeres. En cuanto a los años de experiencia en el ámbito educativo, dividimos a los participantes en cuatro grupos: menos de 5 años (26,5\%), entre 6 y 15 años (28\%), entre 16 y 29 años (25,1\%) y más de 30 años (18,2\%). Un 48,9\%, eran maestros de Educación Infantil, y un porcentaje sensiblemente inferior los titulados en Educación Primaria (15\%), en Educación Especial (5,1\%), alguna del resto de especialidades de maestro: lengua extranjera, música, religión o educación física $(13,8 \%)$ o en otra titulación que imparten su docencia en la etapa de secundaria $(16,7 \%)$. Más de la mitad del profesorado, un $68,7 \%$, desempeñaba su tarea en centros públicos, mientras que un $17,8 \%$ lo hacía en centros concertados y un $13,5 \%$ en centros privados.

Por tratarse de una muestra de conveniencia, hemos analizado la posible existencia de asociaciones entre variables de interés que pudieran estar afectando a los resultados. Así, el análisis de la asociación entre titulación y género no reveló asociaciones significativas. Sin embargo, se encontraron asociaciones significativas entre género y años de experiencia (Chi cuadrado=12,044; $g l=3 ; p=0,009$ ), donde los análisis pusieron de manifiesto que es más probable que las mujeres lleven entre 6 y 15 años, y que los hombres lleven más de 30 años. Por otro lado, el análisis entre titularidad del centro (público, privado o concertado) y 
formación de los docentes resultó significativo (Chi cuadrado=41,184; $g \mid=8 ; p=0,000$ ), y el análisis de los residuos tipificados reveló que existe una mayor probabilidad de que los profesores de centros privados correspondan a la especialidad de Educación Infantil, mientras que en los centros concertados existe una mayor probabilidad de que hayan cursado Educación Especial.

Por otro lado, el análisis de la asociación entre años de experiencia y especialidad también reveló asociaciones significativas (Chi cuadrado=22,236; gl=12; $\mathrm{p}=0,034$ ): los análisis pusieron de manifiesto una mayor probabilidad de que los docentes con una experiencia entre 6 y 15 años hayan cursado Educación Especial.

\section{Instrumento}

Para esta investigación se utilizó un instrumento de evaluación elaborado ad hoc: el Cuestionario de evaluación de la formación docente para la inclusión- CEFI (González-Gil, Martín-Pastor, Flores, Jenaro, Poy, Gómez-Vela y Caballo, 2011). Los ítems del instrumento fueron obtenidos a partir de una exhaustiva revisión bibliográfica sobre el tema y de la realización de dos grupos focales con profesionales de la educación, con una experiencia laboral de entre 7 y 17 años. Se realizó además una aplicación piloto del mismo que permitió comprobar la adecuación de la formulación de cada ítem y elaborar la versión definitiva del CEFI. Así pues el cuestionario está formado por 80 items que se agrupan en 10 subfactores, pertenecientes a las tres dimensiones del Index for Inclusion: culturas, políticas y prácticas. En la Tabla I se presentan dichas dimensiones con los factores correspondientes del cuestionario. Se indica entre paréntesis el número de ítems relacionado con cada factor. El formato de respuesta es tipo Likert de 4 opciones (desde 1- totalmente en desacuerdo a 4totalmente de acuerdo)

Tabla 1.

Dimensiones y factores del CEFI

\begin{tabular}{|l|l|}
\hline \multicolumn{1}{|c|}{ Dimensiones } & \multicolumn{1}{c|}{ Factores } \\
\hline \multirow{3}{*}{ Crear CULTURAS inclusivas } & $\begin{array}{l}\text { Concepción de la diversidad y la educación (16 ítems) } \\
\text { Trabajo colaborativo (4 ítems) } \\
\text { Participación de la comunidad (7 ítems) }\end{array}$ \\
\hline \multirow{3}{*}{ Desarrollar PRÁCTICAS inclusivas POLITICAS inclusivas } & $\begin{array}{l}\text { Política educativa (10 ítems) } \\
\text { Formación profesorado (7 ítems) } \\
\text { Liderazgo (5 ítems) } \\
\text { Recursos y apoyos (10 ítems) }\end{array}$ \\
\hline & $\begin{array}{l}\text { Organización y funcionamiento de la escuela y el aula (8 ítems) } \\
\text { Currículum (7 ítems) }\end{array}$ \\
\hline
\end{tabular}

Las puntuaciones en cada una de las dimensiones se obtienen calculando la media de las respuestas de los ítems que las conforman. Puntuaciones elevadas son indicativas de menores necesidades formativas y actitudes inclusivas positivas, mientras que bajas puntuaciones apuntan hacia mayores necesidades docentes y actitudes negativas hacia la inclusión. Una vez invertidos los ítems con valencia negativa, verificamos la fiabilidad del cuestionario mediante el Coeficiente de Consistencia Interna alfa de Cronbach. Obtuvimos una fiabilidad general de 0,850 para la escala considerada globalmente, y unos índices de consistencia interna de 0,764 para la dimensión de Culturas; de 0,814 para Políticas y de 0,798 para Prácticas. Estos resultados avalan la fiabilidad del instrumento. 


\section{Procedimiento}

Para la selección de la muestra, se estableció, por correo electrónico, contacto con los centros educativos de Castilla y León para informarles sobre los objetivos de la investigación, y solicitar su participación en el estudio. Posteriormente, se concertó una entrevista con el equipo directivo de los centros que contestaron, para resolver dudas relativas al trabajo que se iba a desarrollar y, una vez autorizado, se procedió a organizar la administración del cuestionario, ofreciendo la posibilidad de cumplimentarlo en versión online o en papel. Con la información recopilada hemos realizado constrastes bivariados (análisis de varianza) para identificar en qué factores existían diferencias significativas en función de las distintas variables (género, formación en educación, años de experiencia docente y titularidad de centro), así como análisis post hoc (Scheffé) para determinar qué grupos diferían significativamente.

\section{Resultados}

A fin de realizar un primer acercamiento a la valoración que los profesionales de la educación hacen de su propia formación docente y cómo se sitúan ante el paradigma de la inclusión, en la Tabla II se presentan los resultados obtenidos en las tres dimensiones del cuestionario. Se puede observar que la media en todas ellas es superior a 3, siendo la más elevada la de crear culturas inclusivas $(3,35)$, mientras que la peor puntuación se sitúa en desarrollar prácticas inclusivas. Ello nos permite adelantar la existencia de actitudes bastante favorables hacia la diversidad, pero cierto desconocimiento en cuanto a cómo organizar la respuesta educativa a las necesidades que de ella se derivan.

Tabla 2.

Análisis descriptivos de las puntuaciones en las dimensiones del cuestionario

\begin{tabular}{|l|l|l|l|l|r|}
\hline & $\mathbf{N}$ & Min & Max & Media & DT \\
\hline CULTURAS & 402 & 1,92 & 3,85 & 3,35 &, 24 \\
\hline POLÍTICAS & 402 & 2 & 3,95 & 3,33 &, 30 \\
\hline PRÁCTICAS & 402 & 2,25 & 3,75 & 3,03 &, 27 \\
\hline
\end{tabular}

A continuación y para contrastar las hipótesis se analizaron las posibles diferencias en las puntuaciones obtenidas en función del género. Como se muestra en la Tabla III, únicamente existen diferencias significativas en la dimensión de políticas inclusivas siendo los hombres quienes ofrecen puntuaciones superiores.

Tabla 3.

Estadísticos descriptivos y significación de las diferencias en las puntuaciones (Anova), en función del género

\begin{tabular}{|c|c|c|c|c|c|}
\hline & $\mathbf{N}$ & Media & DT & $\mathbf{F}$ & Sig. \\
\hline CULTURAS & & & & \multirow[t]{3}{*}{0,92} & \multirow[t]{3}{*}{,762 } \\
\hline Hombre & 136 & 3,35 & ,25 & & \\
\hline Mujer & 239 & 3,36 &, 23 & & \\
\hline POLÍTICAS & & & & \multirow[t]{3}{*}{4,185} & \multirow[t]{3}{*}{,041 } \\
\hline Hombre & 136 & 3,38 &, 32 & & \\
\hline Mujer & 239 & 3,31 & ,28 & & \\
\hline PRÁCTICAS & & & & \multirow[t]{3}{*}{,630 } & \multirow[t]{3}{*}{,428 } \\
\hline Hombre & 136 & 3,05 & ,26 & & \\
\hline Mujer & 239 & 3,03 & ,28 & & \\
\hline
\end{tabular}


En segundo lugar se analizaron las posibles diferencias en función de la formación de los maestros. Como se observa en la Tabla IV, se han obtenido diferencias significativas en culturas y prácticas inclusivas. Los análisis post hoc (Scheffé) revelaron que los profesores de Educación Especial obtuvieron puntuaciones significativamente más elevadas que el resto de los grupos.

Tabla 4.

Estadísticos descriptivos y significación de las diferencias en las puntuaciones (Anova), en función de la formación

\begin{tabular}{|c|c|c|c|c|c|}
\hline & $\mathbf{N}$ & Media & DT & $F$ & Sig. \\
\hline $\begin{array}{l}\text { CULTURAS } \\
\text { Maestro } \\
\text { Infantil } \\
\text { Maestro } \\
\text { Primaria } \\
\text { Maestro } \\
\text { especialidad } \\
\text { Educación } \\
\text { Especial } \\
\text { Otra }\end{array}$ & $\begin{array}{l}173 \\
53 \\
49 \\
18 \\
59\end{array}$ & $\begin{array}{l}3,41 \\
3,26 \\
3,34 \\
3,57 \\
3,29\end{array}$ & $\begin{array}{l}21 \\
, 29 \\
, 20 \\
, 13 \\
, 26\end{array}$ & 9,532 & ,000 \\
\hline $\begin{array}{l}\text { POLÍTICAS } \\
\text { Maestro } \\
\text { Infantil } \\
\text { Maestro } \\
\text { Primaria } \\
\text { Maestro } \\
\text { especialidad } \\
\text { Educación } \\
\text { Especial } \\
\text { Otra } \\
\end{array}$ & $\begin{array}{l}173 \\
53 \\
49 \\
18 \\
59\end{array}$ & $\begin{array}{l}3,36 \\
3,24 \\
3,31 \\
3,40 \\
3,28\end{array}$ & $\begin{array}{l}26 \\
, 33 \\
, 28 \\
, 23 \\
\text {, 40 }\end{array}$ & 2,276 & ,061 \\
\hline $\begin{array}{l}\text { PRÁCTICAS } \\
\text { Maestro } \\
\text { Infantil } \\
\text { Maestro } \\
\text { Primaria } \\
\text { Maestro } \\
\text { especialidad } \\
\text { Educación } \\
\text { Especial } \\
\text { Otra }\end{array}$ & $\begin{array}{l}173 \\
53 \\
49 \\
18 \\
59\end{array}$ & $\begin{array}{l}3,05 \\
2,99 \\
3,04 \\
3,33 \\
2,99\end{array}$ & $\begin{array}{l}21 \\
28 \\
, 32 \\
, 21 \\
, 36\end{array}$ & 6,301 & ,000 \\
\hline
\end{tabular}

El análisis de las posibles diferencias en función de los años de experiencia docente puso de manifiesto la existencia de diferencias significativas en políticas inclusivas. Los análisis post hoc revelaron que los docentes con una experiencia entre 6 y 15 años puntuaron significativamente más bajo en esta dimensión. 
Tabla 5.

Estadísticos descriptivos y significación de las diferencias en las puntuaciones (Anova), en función de los años de experiencia docente

\begin{tabular}{|c|c|c|c|c|c|}
\hline & $\mathbf{N}$ & Media & DT & $\mathbf{F}$ & Sig. \\
\hline CULTURAS & & & & \multirow[t]{8}{*}{2,235} & \multirow[t]{8}{*}{,065 } \\
\hline Menos de 5 & 92 & 3,37 & ,25 & & \\
\hline & 97 & 3,33 & ,24 & & \\
\hline Entre 6 y 15 & 87 & 3,35 & ,23 & & \\
\hline años & \multirow[t]{4}{*}{63} & \multirow[t]{4}{*}{3,36} & \multirow[t]{4}{*}{,21 } & & \\
\hline Entre 16 y 29 & & & & & \\
\hline años & & & & & \\
\hline $\begin{array}{l}\text { Más de } 30 \\
\text { años }\end{array}$ & & & & & \\
\hline POLÍTICAS & \multirow{8}{*}{$\begin{array}{l}92 \\
97 \\
87 \\
63\end{array}$} & \multirow{8}{*}{$\begin{array}{l}3,31 \\
3,26 \\
3,38 \\
3,40\end{array}$} & \multirow{8}{*}{$\begin{array}{l}, 29 \\
, 27 \\
30 \\
, 34\end{array}$} & \multirow[t]{8}{*}{2,916} & \multirow[t]{8}{*}{,021 } \\
\hline Menos de & & & & & \\
\hline años & & & & & \\
\hline Entre 6 y 15 & & & & & \\
\hline años & & & & & \\
\hline Entre 16 y 29 & & & & & \\
\hline años & & & & & \\
\hline $\begin{array}{l}\text { Más de } 30 \\
\text { años }\end{array}$ & & & & & \\
\hline PRÁCTICAS & \multirow{7}{*}{$\begin{array}{l}92 \\
97 \\
87 \\
63\end{array}$} & \multirow{7}{*}{$\begin{array}{l}3,07 \\
3,03 \\
2,99 \\
2,99\end{array}$} & & \multirow[t]{7}{*}{1,716} & \multirow[t]{7}{*}{, 140 } \\
\hline Menos de & & & & & \\
\hline años & & & & & \\
\hline Entre 6 y 15 & & & & & \\
\hline años & & & & & \\
\hline $\begin{array}{l}\text { Entre } 16 \text { y } 29 \\
\text { años }\end{array}$ & & & & & \\
\hline $\begin{array}{l}\text { Más de } 30 \\
\text { años }\end{array}$ & & & & & \\
\hline
\end{tabular}

Por último, el análisis de posibles diferencias en función de la titularidad del centro indicó la existencia de diferencias significativas en todas las dimensiones y en el total de la escala. Los análisis post hoc indicaron que, respecto a las Culturas y a las Políticas los docentes de centros públicos ofrecieron valoraciones significativamente más bajas que los pertenecientes a centros concertados. En cuanto a las Prácticas, los centros privados obtuvieron puntuaciones significativamente más bajas que los pertenecientes a centros concertados. 
Tabla 6.

Estadísticos descriptivos y significación de las diferencias en las puntuaciones (Anova), en función de la titularidad del centro

\begin{tabular}{|l|l|l|l|l|l|}
\hline & N & Media & DT & F & Sig. \\
\hline CULTURAS & & & & 4,669 &, 010 \\
Público & 274 & 3,33 &, 24 & & \\
Concertado & 71 & 3,42 &, 23 & & \\
Privado & 54 & 3,39 &, 22 & &, 044 \\
\hline POLítICAS & & & & 3,149 & \\
Público & 274 & 3,31 &, 32 & &, 000 \\
Concertado & 71 & 3,39 &, 28 & & \\
Privado & 54 & 3,38 &, 23 & & \\
\hline PRÁcTICAS & & & & 17,479 & \\
Público & 274 & 3 &, 25 & & \\
Concertado & 71 & 3,20 &, 30 & & \\
Privado & 54 & 2,98 &, 26 & & \\
\hline
\end{tabular}

\section{Discusión y conclusiones}

Con este estudio hemos pretendido ofrecer evidencias adicionales sobre la situación de la inclusión en los centros educativos, a partir de las valoraciones de los propios docentes. Tal y como reflejan los resultados, la dimensión que ofrece inferiores valoraciones se relaciona con el desarrollo de prácticas inclusivas, mientras que la relativa a crear culturas inclusivas obtuvo la puntuación más elevada. Tjernberg y Mattson (2014) ya abundaron en este aspecto, al destacar la importancia de crear una cultura escolar en la que los estudiantes se sientan competentes, valorados y no excluídos. En relación con ello, investigaciones previas (Aguado et ál., 2008; Alemany y Villuendas, 2004; Almeida y Albert, 2009; Álvarez et ál., 2005; Avramidis y Kalyra, 2007; Chiner, 2011; Lledó y Arnáiz, 2010; López López y Hinojosa, 2012; Vigo y Soriano, 2014) señalan que la mayoría de los profesores son partidarios de la inclusión educativa. De hecho, manifiestan tener una buena actitud y expectativas positivas hacia sus alumnos, independientemente de cuáles sean sus características. Sin embargo, no saben o se muestran más resistentes a la hora de modificar su práctica educativa para que ésta se rija por los parámetros de la inclusión. Apelan a dificultades de índole organizativo y pedagógico como la escasez de recursos y de tiempo, el escaso apoyo de la administración educativa y de las familias, o la falta de formación específica. En este sentido, Torres y Fernández (2015) se cuestionan si, a pesar de la evolución terminológica, realmente se ha producido un cambio en el quehacer profesional. Recientes estudios indican que el $88 \%$ de los docentes casi nunca adapta las actividades en función de las necesidades del alumnado, realizan pocos cambios y éstos son poco significativos (Chiner, 2011). A ello hay que añadir que, tal y como señalan Almeida y Albert (2009), Colmenero (2009) o Sapon Shevin (2013), se tiende a relacionar la atención a la diversidad únicamente con los escolares identificados con necesidades específicas de apoyo educativo y no con un modelo educativo dirigido a todos los estudiantes, con independencia de sus necesidades o características.

En lo que respecta a la influencia de la variable género, los resultados desvelaron que los hombres valoraban las políticas de un modo significativamente más elevado. Si bien algunos estudios han obtenido resultados similares (p.e. Batsiou, Bebetsos, Panteli y Antoniou, 2008) otros indican que son las mujeres quienes ofrecen valoraciones más altas (Chiner, 2011), e incluso algunos autores no encuentran diferencias entre géneros (p.e. 
Almeida y Alberte, 2009). Si bien hay trabajos que atribuyen estos resultados a la desigual distribución del género en los diferentes niveles formativos, con una mayor representación de mujeres en las etapas infantiles (Chiner, 2011, Jurado de los Santos y Olmos Rueda, 2010), en el presente estudio el género se distribuía homogéneamente en las especialidades y por tanto, en los niveles formativos en los que se imparte la docencia. No obstante, el hecho de que exista una desigual distribución de hombres y mujeres en cuanto a los años de experiencia, con una mayor antigüedad en el caso de los hombres y con un mayor predominio de mujeres con una trayectoria entre 6 y 15 años, pudiera contribuir a explicar estos resultados.

En cuanto a posibles diferencias en las puntuaciones de los participantes según su formación inicial los maestros de educación especial consiguieron puntuaciones más elevadas que el resto de sus compañeros en culturas y prácticas. Estos resultados coinciden con estudios previos (Alemany y Villuendas, 2004; Almeida y Albert, 2009; Avramidis y Kalyra, 2007; Chiner, 2011; Lledó y Arnáiz, 2010; Sánchez Palomino, 2007) en los que se pone de manifiesto que los profesores de educación especial tienden a tener una visión más positiva de la inclusión, sintiéndose más competentes y mejor preparados para responder a las necesidades específicas de los alumnos, atribuyendo, en parte, lo anterior a su formación especializada en este campo. Por el contrario, estudios realizados con estudiantes del antiguo curso de aptitud pedagógica indican que casi la totalidad consideraban que la inclusión de los alumnos con necesidades educativas especiales es tarea de los especialistas, y que el profesor del aula ordinaria no estaba preparado para asumir el reto de la educación para todos (Sánchez Palomino, 2007). Por otro lado, algunos estudios (Chiner, 2011; Jurado de los Santos y Olmos Rueda, 2010) afirman que el profesorado de educación infantil y primaria muestra actitudes más favorables que el de secundaria al disponer de más recursos y apoyos para funcionar dentro de un modelo de escuela inclusiva. A ello los autores también añaden como posible justificación de esta situación, la menor formación didáctica en general y en atención a la diversidad en particular que posee el profesorado de educación secundaria obligatoria.

Respecto a la influencia de los años de trayectoria profesional los resultados sugieren que la experiencia docente establecía diferencias significativas a favor de una mayor antigüedad en lo relativo a políticas inclusivas. Sin embargo, son los profesores con menos años trabajados los que mejor puntuaron en culturas y prácticas, aunque no fuera de manera significativa. Desde nuestro punto de vista, y coincidiendo con Álvarez et ál. (2008), lo anterior se puede deber a una relación entre una visión negativa de la educación dentro de una línea inclusiva y los años de antigüedad docente. Ésto en el sentido de que, si bien es cierto que la experiencia te proporciona sabiduría y mayores oportunidades de aprendizaje, hay profesores que en esta situación se sienten cansados, reacios al cambio y muy distanciados de la evolución que se ha producido en el contexto educativo respecto a la atención a los alumnos con necesidades educativas especiales y la organización de la misma dentro de un modelo inclusivo, manifestando incluso mayor hostilidad ante dicho modelo que sus compañeros (Soodak, Podell y Lehman, 1998, citados por Chiner, 2011; Colmenero, 2009). Sin embargo, el estudio de Chiner (2011) contradice lo anterior al identificar la variable experiencia profesional como factor positivo que influye en una mayor consecución de una verdadera educación para todos al haber tenido más oportunidades para aprender, interiorizar y desarrollar prácticas inclusivas.

Por último, al analizar la influencia de la titularidad del centro, los resultados indican que los participantes de centros concertados obtienen puntuaciones más elevadas que los de centros públicos en cuanto a cultura y políticas, y más elevadas que los centros privados en cuanto a prácticas. Como señalamos en el apartado de participantes, existe una mayor 
presencia de profesores con la especialidad de Educación Especial en centros concertados, lo que podría explicar estos resultados. Investigaciones adicionales en esta línea contribuirían a esclarecer el peso de estas variables y así arrojar luz sobre la diversidad de resultados hallada en la literatura sobre el tema (Álvarez et al., 2008; Jurado de los Santos y Olmos Rueda, 2010; Lledó y Arnáiz, 2010).

Si bien el presente estudio presenta una serie de limitaciones, como la relacionada con el procedimiento de selección de los participantes, lo que condiciona las posibilidades de generalizar los presentes hallazgos, los resultados ponen de manifiesto la necesidad de seguir promoviendo prácticas inclusivas, precedidas de un consenso sobre políticas y culturas que favorezcan dicha inclusión. Como profesionales implicados en la formación de maestros, pedagogos y psicólogos es nuestra responsabilidad analizar críticamente la formación que estamos proporcionando, el enfoque que adoptamos y en consecuencia, las políticas que apoyamos, las culturas que promovemos y las prácticas que fomentamos y enseñamos a nuestros estudiantes, porque todo ello, como hemos evidenciado, tiene su reflejo en las políticas, culturas y prácticas que éstos promueven posteriormente en su vida profesional.

\section{Referencias bibliográficas}

Acedo, C. (2011). Preparing teachers for inclusive education. Prospects, 41, 301-302.

Aguado, A. L., Alcedo, M. Á. y Árias, B. (2008). Cambio de actitudes hacia la discapacidad con escolares de primaria. Psicothema, 20 (4), 697-704.

Ahmmed, M., Sharma, U. y Deppeler, J. (2014) Variables affecting teachers' intentions to include students with disabilities in regular primary schools in Bangladesh. Disability \& Society, 29 (2), 317-331.

Alemany, I. y Villuendas, M. D. (2004). Las actitudes del profesorado hacia el alumnado con necesidades educativas especiales. Convergencia. Revista de Ciencias Sociales, 11 (34), 183-215.

Almeida, M. S. y Albert, J. R. (2009). Las concepciones de los profesores y la respuesta a la inclusión en Lisboa. Revista de Educación Inclusiva, 2 (2). Recuperado de http://www.ujaen.es/revista/rei/linked/documentos/documentos/3-2.pdf

Álvarez, E., Álvarez, M., Castro, O., Campo, A. y Fueyo, E. (2008). Funcionamiento de la integración en la enseñanza secundaria obligatoria según la percepción del profesorado. Psicothema, 20 (1), 56-62.

Álvarez, M., Castro, P., Campo, M. A. y Álvarez, E. (2005). Actitudes de los maestros ante las necesidades educativas específicas. Psicothema, 17 (4), 601-606.

Avramidis, E. y Kalyra, E. (2007). The influence of teaching experience and professional development on greek teachers 'attitudes towards inclusion. European Journal of Special Needs Education, 22 (4), 367-389.

Batsiou, S., Bebetsos, E. Panteli, P. y Antoniou, P. (2008). Attitudes and intention of greek and cypriot primary education teachers towards teaching pupils with special Education needs in mainstream schools. International Journal of Inclusive Education, 21 (2), 201-219

Blanco, R. (2008). Construyendo las bases de la inclusión y la calidad de la educación en la primera infancia. Revista de Educación, 347, 33-54. 
Booth, T. (2009). El uso del Index for Inclusion en Inglaterra. En Giné, C., Durán, D., Font, J. y Miquel, E. (Eds.). La educación inclusiva. De la exclusión a la plena participación de todo el alumnado (pp.143-160). Barcelona: Horsori Editoral.

Booth, T. y Ainscow. M. (2002). Index for Inclusion. Developing learning and participation in schools ( $2^{\mathrm{a}}$ ed.). Manchester: CSIE.

Booth, T. y Ainscow, M. (2011). Index for Inclusion. Developing learning and participation in schools ( $3^{\mathrm{a} e d .}$ ). Manchester: CSIE.

Booth, T., Ainscow, M. y Kingston, D. (2006). Index for Inclusion: developing play, learning and participation in early years and childcare schools. Bristol, UK: CSIE.

Chiner, E. (2011). Las percepciones y actitudes del profesorado hacia la inclusión del alumnado con necesidades educativas especiales como indicadores del uso de prácticas inclusivas en el aula. (Tesis inédita de Doctorado). Universidad de Alicante.

Colmenero, M. J. (2009). Influencia y repercusión de la experiencia como docente en la atención a la diversidad. Su incidencia en la formación. Revista de Educación Inclusiva, 2 (3), 71-82.

Deutsch, D. y Chowdhuri, N. (2011). Effective inclusive education: Equipping education professionals with necessary skills and knowledge. Prospects, 41, 323-339.

Donnelly, V. y Watkins, A. (2011). Teacher education for inclusion in Europe. Prospects, 41, 341-353.

Durán, D., Echeita, G., Giné, C., Miquel, E., Ruiz, C. y Sandoval, M. (2005). Primeras experiencias del uso de la guía para la evaluación y mejora de la educación inclusiva (Index for Inclusion) en el Estado Español. REICE. Revista Electrónica Iberoamericana sobre Calidad, Eficacia y Cambio en Educación, 3 (1), 464-467.

Echeita, G, Verdugo, M.A., Sandoval, M., Simón, C., López, M. González-Gil, F. et ál. (2008). La opinión de FEAPS sobre el proceso de inclusión educativa. Siglo Cero 39 (4), 2650.

Echeita, G. (2007). Educación para la inclusión. Educación sin exclusiones. Madrid: Narcea.

Escudero, J. M. y Martínez, B. (2012). Las políticas de lucha contra el fracaso escolar: ¿programas especiales o cambios profundos del sistema y la educación? Revista de Educación, número extraordinario, 174-193.

González, M.T. (2008). Diversidad e Inclusión Educativa: Algunas Reflexiones sobre el Liderazgo en el Centro Escolar. REICE-Revista Electrónica Iberoamericana sobre Calidad, Eficacia y Cambio en Educación, 6 (2), 82-99.

González-Gil, F., Gómez-Vela, M. y Jenaro, C. (2007). Index para la Inclusión: Desarrollo del juego, el aprendizaje y la participación en Educación Infantil. Salamanca: Universidad de Salamanca.

Göransson, K. y Nilholm, C. (2014). Conceptual diversities and empirical shortcomings - a critical analysis of research on inclusive education. European Journal of Special Needs Education, 23 (3), 265-280

Jurado de los Santos, P. y Olmos Rueda, P. (2010). Las actitudes del profesorado. Eje clave para la intervención educativa inclusiva. En Rojas, S., García Lastra, M., Calvo, A., Lázaro, S., Haya, I., Ruiz, J. et ál. (Coords.). Actas del Congreso Internacional "La educación inclusiva hoy: escenarios y protagonistas" y XXVII Jornadas Nacionales de Universidades y Educación Especial (227-241). Universidad de Cantabria. 
Kyriazopoulou, M. y Weber, H. (Eds.). (2009). Desarrollo de indicadores sobre educación inclusiva en Europa. Odense, Denmark: European Agency for Development in Special Needs Education.

Lledó, A. y Arnaiz, P. (2010). Evaluación de las prácticas educativas del profesorado de los centros escolares: Indicadores de mejora desde la educación inclusiva. Revista Iberoamericana sobre Calidad, Eficacia y Cambio en Educación, 8 (5), 96-109.

López, A. L., Durán, D., Echeita, G., Giné, C., Miquel, E. y Sandoval, M. (2002). Guía para la evaluación y mejora de la educación inclusiva. Madrid: Consorcio Universitario para la Educación Inclusiva.

López López, M. C. y Hinojosa, E. F. (2012). El estudio de las creencias sobre la diversidad cultural como referente para la mejora de la formación docente. Educación $X X_{1}, 15$ (1), $195-218$.

López Melero, M. (2012). La escuela inclusiva: una oportunidad para humanizarnos. Revista Interuniversitaria de Formación de Profesorado, 26 (2), 131-160

Mckinsey and Company (2007). How the world's best performing school systems come out on top. Recuperado de http://www.mckinsey.com/locations/UK_Ireland/*/media/Reports/UKI/Education_re port.ashx

Miquel, E. (2009). El uso del Index for Inclusion para cambiar las prácticas educativas en los centros de Catalunya. En Giné, C., Durán, D., Font, J. y Miquel, E. (Eds.). La educación inclusiva. De la exclusión a la plena participación de todo el alumnado (pp.127-142). Barcelona: Horsori Editoral.

Molina, S. y Holland, Ch. (2010). Educación especial e inclusión: aportaciones desde la investigación. Revista Educación y Pedagogía, 22 (56), 31-43.

Mosia, P.A. (2014) Threats to inclusive education in Lesotho: An overview of policy and implementation challenges. Africa Education Review, 11 (3), 292-310.

Parrilla, A. (2007). Inclusive Education in Spain: a view from inside. En Barton, L. y Amstrong, F. (Eds.). Policy, Experience and Change: Cross Cultural Reflections on Inclusive Education (pp. 19-36). Dordrecht: Springer.

Sánchez Palomino, A. (2007). Investigación sobre la formación inicial del profesorado de educación secundaria para la atención educativa a los estudiantes con necesidades especiales. Revista Interuniversitaria de Formación de Profesorado, 21 (2/3), 149-181.

Sapon-Shevin, M. (2013). La inclusión real: Una perspectiva de justicia social. Revista de Investigación en Educación, 11 (3), 71-85.

Tjernberg, C. y Mattson, E.H. (2014). Inclusion in practice: a matter of school culture. European Journal of Special Needs Education, 29 (2), 247-256.

Torres, J. A. y Fernández, J.M. (2015). Promoviendo escuelas inclusivas: análisis de las percepciones y necesidades del profesorado desde una perspectiva organizativa, curricular y de desarrollo profesional. Revista Electrónica Interuniversitaria de Formación del Profesorado, 18 (1), 177-200

Vigo, B. y Soriano, J. (2014). Teaching practices and teachers' perceptions of group creative practices in inclusive rural schools. Ethnography and Education, 9 (3), 253-269.

Vlachou, A. (2004). Education and inclusive policy-making: implications for research and practice. International Journal of Inclusive Education, 8 (1), 3-21. 
Weiß, S., Kollmannsberger, M., Lerche, T., Oubaid, V. y Kiel, E. (2014). The pedagogic signature of special needs education. European Journal of Special Needs Education, 29 (2), 200-219. 